VANESSA MORAIS LIMA

\title{
PAPEL DA MUTAÇÃO ALDH2*2 NA INSUFICIÊNCIA CARDÍACA: POTENCIAL TERAPÊUTICO DA ALDA-1
}

Tese apresentada ao Programa de Pós-Graduação em Ciências Morfofuncionais do Instituto de Ciências Biomédicas da Universidade de São Paulo, para obtenção do Título de Doutor em Ciências.

Área de concentração: Ciências Morfofuncionais.

Orientador: Prof. Dr. Julio Cesar Batista Ferreira.

Versão corrigida. A versão original eletrônica encontra-se disponível tanto na Biblioteca do ICB quanto na Biblioteca Digital de Teses e Dissertações da USP (BDTD).

São Paulo

2016 


\section{RESUMO}

Lima, VM. Papel da mutação $A L D H 2{ }^{*} 2$ na insuficiência cardíaca: potencial terapêutico da Alda-1. [Tese (Doutorado em Ciências Morfofuncionais)]. São Paulo: Instituto de Ciências Biomédicas, Universidade de São Paulo; 2016.

A enzima aldeído desidrogenase 2 (ALDH2), localizada na matriz mitocondrial, é crucial na manutenção da viabilidade celular. Seu principal papel é metabolizar aldeídos reativos produzidos durante 0 estresse oxidativo. Recentemente demonstramos que a inibição farmacológica da ALDH2 resulta em acúmulo de aldeídos citotóxicos e maior dano ao miocárdio frente estresse isquêmico. Atualmente, estima-se que $8 \%$ da população mundial ( 600 milhões de pessoas) apresentam uma mutação pontual no gene da ALDH2 (E487K) que confere perda de até $95 \%$ na sua atividade enzimática. Baseado nisso, o nosso objetivo foi avaliar o impacto da redução da atividade catalítica da ALDH2 decorrente da mutação E487K no metabolismo cardíaco basal e com insuficiência cardíaca induzida por infarto do miocárdio; e o papel da ativação sustentada da ALDH2 com a Alda-1 sobre esse fenótipo. Para isso, avaliamos o consumo de oxigênio e liberação de $\mathrm{H}_{2} \mathrm{O}_{2}$ em mitocôndrias cardíacas isoladas de camundongos selvagens e ALDH2*2 mutantes (knock-in portadores da mutação E487K no gene da ALDH2) em heterozigose e homozigose. Ainda, avaliamos, por ecocardiografia, se os animais mutantes são mais suscetíveis aos danos metabólicos oriundos do infarto do miocárdio. Em nossos resultados, observamos que os animais mutantes possuem a atividade e a expressão proteica da ALDH2 reduzidas comparadas ao WT, 44\% no heterozigoto e $75 \%$ no homozigoto. Vimos também, que os animais com a mutação ALDH2*2, tanto em heterozigose, quanto em homozigose, desenvolvem disfunção cardíaca e remodelamento ventricular equivalente ao animal selvagem após infarto do miocárdio. Porém, os animais com a mutação E487K possuem uma significante redução no consumo de oxigênio máximo (medido indiretamente em teste físico até a exaustão) e em mitocôndria cardíaca isolada, quando comparados aos animais selvagens dentro do grupo controle. Entretanto, a mitocôndria desses animais não parece estar disfuncional, respondendo com menor liberação de peróxido de hidrogênio e maior consumo de $\mathrm{O}_{2}$ que os animais WT quando infartados. Além disso, o tratamento sustentado com a molécula ativadora específica da ALDH2 (Alda-1) durante 6 semanas aumenta a atividade da ALDH2, que remove de forma mais eficiente os aldeídos tóxicos, restaurando a função cardíaca dos animais infartados, independente do genótipo. Baseado nisso, nossos resultados fornecem evidências de que a ALDH2 é uma importante enzima que auxilia na manutenção da viabilidade cardíaca pós-infarto do miocárdio, e que a redução da carga de aldeídos cardíacos através da ativação seletiva da ALDH2 é suficiente para atenuar danos causados pela insuficiência cardíaca.

Palavras-chave: ALDH2. Mutação ALDH2*2. Insuficiência cardíaca. Metabolismo mitocondrial. Alda-1. 


\begin{abstract}
Lima, VM. Role of ALDH2*2 mutation in heart failure: therapeutic potencial of Alda-1. [Ph.D. Thesis (Morphofunctional Sciences)]. São Paulo: Instituto de Ciências Biomédicas, Universidade de São Paulo; 2016.

Aldehyde dehydrogenase 2 (ALDH2), located in the mitochondrial matrix, is critical for the maintenance of cellular redox balance by removing reactive aldehydes produced during oxidative stress. We recently reported that pharmacological inhibition of ALDH2 results in accumulation of cytotoxic aldehydes and increased myocardial damage during ischemic stress. Currently, it is estimated that $8 \%$ of the world population have a point mutation in the ALDH2 gene (E487K) which reduces its enzymatic activity by $95 \%$. Based on this we aim to assess the impact of the E487K variant of ALDH2 on cardiac mitochondrial metabolism during myocardial infarctioninduced heart failure; and the role of the sustained activation of ALDH2 using Alda-1. We evaluated the oxygen consumption and $\mathrm{H}_{2} \mathrm{O}_{2}$ release using cardiac mitochondria isolates from wild-type (WT), heterozygous and homozygous ALDH2 E487K knock-in mice. We also evaluated whether ALDH2 E487K knock-in mice are more prone to develop cardiac dysfunction after myocardial infarction. Our results show that mice carrying the ALDH2 variant have reduced ALDH2 activity and protein levels compared to WT mice. Despite of reduced oxidative metabolism, animals with ALDH2 mutation develop cardiac dysfunction and ventricular remodeling equivalent to WT animals after myocardial infarction. Finally, sustained Alda- 1 treatment (ALDH2 activator) during 6 weeks improved cardiac function of infarcted animals, regardless of genotype. Alda-1 treatment was effective in normalizing both mitochondrial respiration and reactive oxygen species in WT animals after cardiac dysfunction, with no impact on mutant mice. In conclusion, our findings unravel a critical role for ALDH2 activation against myocardial infarction-induced ventricular dysfunction in mice.
\end{abstract}

Keywords: ALDH2. ALDH2² mutation. Heart failure. Mitochondrial metabolism. Alda-1. 


\section{INTRODUÇÃO}

\subsection{Insuficiência cardíaca}

Segundo a Organização Mundial de Saúde as doenças cardiovasculares são a principal causa de mortes no mundo. A estimativa em 2012 foi de que a cada ano, 17,5 milhões de pessoas morrem por essas doenças, representando $31 \%$ de todas as mortes. Dentre as doenças cardiovasculares, a doença isquêmica cardíaca, como o infarto agudo do miocárdio, é a maior causa de morbidade e mortalidade no mundo (WHO, 2014).

A insuficiência cardíaca (IC) é a via final comum da maioria das doenças cardiovasculares, atingindo mais de 23 milhões de pessoas em todo o mundo, em taxas crescentes a cada ano (Liu, Eisen, 2014). Nos Estados Unidos surgem $550 \mathrm{mil}$ novos casos/ano, sendo que 1 a cada 5 pessoas corre o risco de desenvolver IC (Liu, Eisen, 2014). No Brasil a IC é a causa mais frequente de internação por doenças cardiovasculares e, em 2007 foi responsável pelo gasto de 3\% dos recursos totais destinados à internação do sistema único de saúde (SBC, 2012).

A IC é caracterizada pela incapacidade dos ventrículos em bombear quantidades adequadas de sangue para manter a perfusão tecidual (Colucci, 1998). Essa síndrome era descrita, na década de 70 , como um distúrbio hemodinâmico associado à redução do débito cardíaco, com consequente baixo fluxo renal, levando à retenção de sódio, água e o surgimento de edemas periféricos e pulmonares que acabavam por levar o paciente ao óbito. Atualmente, a IC é vista como uma doença sistêmica. Quando o débito cardíaco é reduzido após agressão miocárdica, sistemas neuro-humorais (sistema nervoso simpático e sistema renina angiotensina) são ativados com 0 objetivo de desencadear respostas compensatórias (taquicardia, aumento da contratilidade miocárdica, hipertrofia cardíaca e remodelamento cardíaco) e preservar a homeostase cardiovascular (Fiorelli et al., 2008; Poyton, Ball, Castello, 2009). Embora originalmente vista como uma resposta compensatória benéfica, a liberação endógena sustentada de neurohormônios parece exercer papel deletério e crucial no estabelecimento/desenvolvimento da IC, pelo aumento da sobrecarga de volume e da pós-carga do ventrículo com contratilidade já diminuída. Além disso, a 
hiperativação neuro-humoral sustentada resulta no aumento do consumo de oxigênio pelo miocárdio e da resistência vascular (Chen, Niki, 2006). Por fim, os neuro-hormônios podem exacerbar as anormalidades metabólicas já existentes, ocasionando o aparecimento de arritmias cardíacas (Liu, O'Rourke, 2009).

Em detrimento a hiperativação de fatores neuro-humorais, a progressão da IC é caracterizada por uma série de anormalidades celulares. Uma das alterações celulares mais marcantes na IC é a disfunção mitocondrial, que é normalmente acompanhada pelo estresse oxidativo e seus efeitos deletérios (Tsutsui, Kinugawa, Matsushima, 2011). Atualmente sabemos que o prejuízo na produção de energia, associado aos efeitos deletérios da exacerbada produção de radicais livres está diretamente relacionado à menor viabilidade cardíaca tanto em modelos animais de IC quanto em pacientes portadores de IC (Abel, Doenst, 2011; Tsutsui, Kinugawa, Matsushima, 2011; Ventura-Clapier et al., 2011).

\subsection{Bioenergética mitocondrial}

A mitocôndria é conhecida como a central energética celular, uma vez que processos como beta-oxidação, Ciclo de Krebs e fosforilação oxidativa ocorrem em seu interior (Mitchell, Moyle, 1967). A mitocôndria também é local de outras importantes reações metabólicas, incluindo o ciclo da ureia, o metabolismo lipídico e a síntese de aminoácidos, hormônios e outras moléculas envolvidas na sinalização intracelular (Brookes et al., 2004). Inclusive, já é sabido, que as mitocôndrias possuem participação na regulação direta de processos como apoptose, diferenciação, crescimento e proliferação celular (Antico Arciuch et al., 2012; Ott et al., 2007).

A síntese de ATP mitocondrial ocorre por meio do acoplamento da fosforilação oxidativa à cadeia de transporte de elétrons. O gradiente eletroquímico de prótons gerado durante o transporte de elétrons através da cadeia de transporte de elétrons é utilizado na conversão de ADP (adenosina difosfato) + Pi (fosfato inorgânico) em ATP pelo complexo ATP-sintase (complexo V) (Mitchell, Moyle, 1967). Praticamente toda a energia disponível a partir da oxidação de carboidratos, gorduras e outros substratos é inicialmente armazenada na forma de elétrons de alta energia, provenientes da dissociação dos átomos de hidrogênio em prótons e 
elétrons, que são transferidos para $0^{\circ} \mathrm{O}_{2}$ através de uma série de complexos proteicos.

A cadeia respiratória transporta elétrons dos complexo I (malato-NADH) ou complexo II (succinato-FADH2) para os complexos III e IV, onde o oxigênio é reduzido a $\mathrm{H}_{2} \mathrm{O}$. Nesse processo, elétrons podem extravasar para o espaço intermembranar mitocondrial e reagir com $\circ \mathrm{O}_{2}$, formando ânion superóxido $\left(\mathrm{O}_{2}{ }^{-}\right)$. Este por ação da superóxido desmutase mitocondrial (MnSOD), que tem como cofator o manganês é transformado em $\mathrm{H}_{2} \mathrm{O}_{2}$. $\mathrm{O} \mathrm{H}_{2} \mathrm{O}_{2}$ pode difundir pela membrana ou se acumular na mitocôndria, onde pode ser transformado em $\mathrm{H}_{2} \mathrm{O}$ e $\mathrm{O}_{2}$ pela ação da catalase ou pela oxidação da glutationa peroxidase/tioredoxina peroxidase, utilizando os estoques de $\mathrm{NAD}(\mathrm{P}) \mathrm{H}$ como fonte de elétrons. Se esse sistema não for eficaz e o $\mathrm{H}_{2} \mathrm{O}_{2}$ se acumular, pode ser gerado o radical hidroxila $(\mathrm{OH})$ que é um dos mais reativos dentre as espécies reativas (Facundo, 2007; Kowaltowski et al., 2009) (Figura 1).

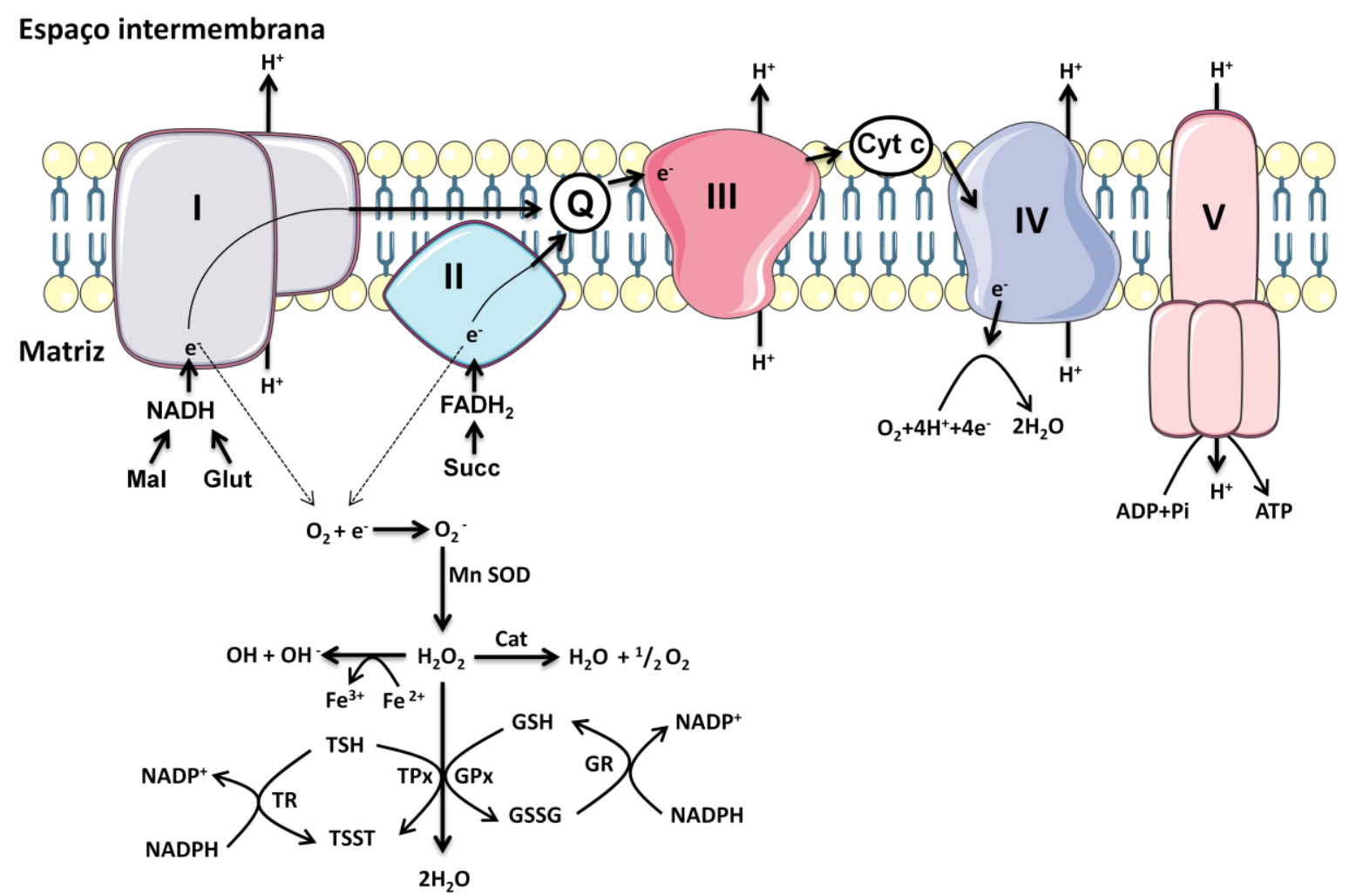

Figura 1 - Cadeia transportadora de elétrons com formação e remoção de EROS. A cadeia respiratória transporta os elétrons dos substratos do complexo I (Mal = Malato e Glut $=$ Glutamato) ou complexo II (Succ = succinato), adquiridos do NADH ou FADH ${ }_{2}$, para a coenzima Q, complexo III, citocromo c (Cyt c) e o complexo IV, onde o oxigênio molecular é reduzido à $\mathrm{H}_{2} \mathrm{O}$. E a energia livre resultante é utilizada para a síntese de ATP, pelo retorno de prótons pelo complexo $\mathrm{V}$. No entanto, os elétrons podem escapar, formando o ânion 
superóxido $\left(\mathrm{O}_{2}^{-}\right)$, este por sua vez, é transformado em $\mathrm{H}_{2} \mathrm{O}_{2}$ pela MnSOD (Superóxido dismutase - cofator manganês). $\mathrm{O} \mathrm{H}_{2} \mathrm{O}_{2}$ pode ser transformado em $\mathrm{H}_{2} \mathrm{O}$ e $\mathrm{O}_{2}$ pela catalase (Cat) ou pela glutationa peroxidase/tiredoxina peroxidase (GPx/TPx) com oxidação da glutationa/tioredoxina (GSH/TSH). A glutationa ou tioredoxina oxidadas (GSSG/TSST) podem ser reconvertidas a GSH/TSH pelas glutationa ou tioredoxina redutases (GR ou TR), que utilizam os estoques de NADPH como fonte de elétrons. $\mathrm{Se} \circ \mathrm{H}_{2} \mathrm{O}_{2}$ não for reduzido à $\mathrm{H}_{2} \mathrm{O}$, ele pode se difundir pela membrana, se acumular na mitocôndria, ou ainda, pode gerar o radical hidroxila $(\mathrm{OH})$ pela reação de Fenton. (Fonte: Lima, 2016; adaptado de Facundo, 2007; Tahara, Navarete, Kowaltowski, 2009)

A produção exacerbada das diversas espécies reativas de oxigênio e nitrogênio decorrente da disfunção mitocondrial tem sido considerada essencial na geração e propagação do dano celular que resulta no estabelecimento e agravamento das doenças cardiovasculares (Palaniyandi et al., 2010).

\subsection{Disfunção mitocondrial na insuficiência cardíaca}

Diversas evidências têm mostrado que a disfunção mitocondrial está relacionada com doenças degenerativas, como Alzheimer, Parkinson (Abramov et al., 2011; Young-Collier, McArdle, Bennett, 2012) e insuficiência cardíaca (Abel, Doenst, 2011; Tsutsui, Kinugawa, Matsushima, 2011; Ventura-Clapier et al., 2011).

No coração, aproximadamente $90 \%$ das espécies reativas de oxigênio são produzidas através da cadeia transportadora de elétrons na mitocôndria (Di Lisa et al., 2009; Poyton, Ball, Castello, 2009). Em condições de homeostase, estas espécies reativas são reduzidas por enzimas ou por "scavengers" como a vitamina E ou ubiquinol. Contudo, em processos de envelhecimento, hipóxia ou hiperativação neuro-humoral, pode ocorrer uma alteração no controle redox, resultando em aumento excessivo nas concentrações de espécies reativas de oxigênio.

Em geral, o aumento exacerbado da produção de espécies reativas de oxigênio pela mitocôndria, resulta em redução da viabilidade celular. Esse fenômeno está diretamente associado à oxidação de DNA mitocondrial, lipídios, açúcares e proteínas, resultando em uma série de anormalidades na mitocôndria, como redução do potencial da membrana interna, diminuição na síntese de ATP, aumento da captação de cálcio, prejuízo no controle de qualidade de proteína e ativação de vias pró-apoptóticas (Chen, Niki, 2006).

Dentre os oxidantes produzidos, encontram-se os peróxidos lipídicos que podem ser metabolizados pela glutationa peroxidase. Contudo, na presença de um doador de elétron, este peróxido sofre uma reação não-enzimática e se divide para 
produzir um aldeído tóxico, o 4-hidroxi-2-nonenal (4-HNE). Esta molécula é gerada endogenamente, a partir da clivagem de hidroperóxidos lipídicos, e é altamente reativa com resíduos de cisteína, lisina ou histidina presentes em proteínas. Resumidamente, o processo da peroxidação lipídica pode ser definido como uma deterioração auto-oxidativa de ácidos graxos poli-insaturados.

É interessante mencionar que, quando produzidos em níveis basais (i.e. $<1$ $\mu \mathrm{M})$, os aldeídos como o 4-HNE, atuam como moléculas sinalizadoras intracelulares. Entretanto, sob condições de estresse oxidativo, ocorre produção exacerbada destes aldeídos, saturando as vias de seu metabolismo, o que favorece modificações de moléculas biológicas e o início de processos patológicos. A importância clínica deste aldeído decorre de sua habilidade em se difundir para locais mais distantes de onde é produzido. Assim, ele pode formar adutos (ligações químicas de alta afinidade) com diversas proteínas, modificando-as irreversivelmente. $\mathrm{O}$ 4-HNE pode se ligar a albumina plasmática, enzimas do metabolismo, proteínas de membrana e citosólicas, cadeia transportadora de elétrons, entre outros (Budas, Disatnik, Mochly-Rosen, 2009; Chen, Sun, MochlyRosen, 2010; Kutuk, Basaga, 2007; Song et al., 2011; Yang et al., 2003). Este aldeído tem sido extensivamente relacionado à etiologia de diversas doenças, como as cardiovasculares e hepáticas (Kaplan et al., 2007).

O 4-HNE possui um carbono reativo, localizado na posição 3 (posição central) da molécula. Este carbono pode formar uma ligação (ligação de Michaelis) com resíduos de cisteína, histidina ou lisina da proteína alvo, modificando-a. Estudos têm demonstrado que cerca de 1 a $8 \%$ do 4-HNE formado na célula modifica proteínas, resultando em perda de função dessas proteínas (Backos, et al., 2011).

Outro tipo de modificação decorrente de ataque eletrofílico é a carbonilação de proteínas (ligação de grupamentos carbonila), um tipo de oxidação proteica que pode ser produzida pela reação direta de espécies reativas de oxigênio com resíduos de arginina, lisina e treonina. As carbonilas podem também ser geradas pela reação de Michaelis, entre resíduos de cisteína, lisina ou histidina e intermediários da peroxidação lipídica, como o 4-HNE (Budas, Disatnik, MochlyRosen, 2009; Chen, Sun, Mochly-Rosen, 2010; Kutuk, Basaga, 2007; Song et al., 2011; Yang et al., 2003). Resultados prévios do nosso grupo mostram que níveis 
elevados de proteínas carboniladas são encontrados no coração de roedores e humanos portadores de IC, assim, a carbonilação é considerada um importante biomarcador na IC (Ferreira et al., 2012).

A disfunção mitocondrial, bem como o desbalanço redox, são processos que colaboram diretamente para o estabelecimento das doenças cardiovasculares. Porém, os mecanismos celulares envolvidos nesses processos ainda são controversos. Demonstramos, em colaboração com a Universidade de Stanford, que a isquemia resulta em acúmulo de 4-HNE cardíaco em decorrência de dois processos: 1) oxidação de lipídios presentes na membrana mitocondrial; e 2) inativação da enzima aldeído desidrogenase 2 (ALDH2), responsável pela remoção do 4-HNE. De fato, Ma e colaboradores (Ma et al., 2011) demonstraram que camundongos que hiperexpressam ALDH2 apresentam níveis reduzidos de 4-HNE após isquemia/reperfusão, já a ablação do gene da ALDH2 resulta em maior acúmulo de 4-HNE frente ao mesmo estresse.

\subsection{Aldeído desidrogenase 2 (ALDH2) e mutação ALDH2*2}

A aldeído desidrogenase $2(\mathrm{ALDH} 2)$ faz parte da superfamília aldeído desidrogenase (ALDH), composta por enzimas que catalisam a oxidação de aldeídos a seus ácidos correspondentes, não tóxicos, em uma reação irreversível dependente de $\operatorname{NAD}(P)^{+}$. Já foram descritos 19 genes que codificam a superfamília ALDH, localizados em cromossomos diferentes. As ALDHs são conservadas na evolução e estão distribuídas em várias regiões subcelulares incluindo citosol, mitocôndria, retículo endoplasmático e núcleo. Além disso, as ALDHs possuem uma ampla distribuição tecidual e exibem especificidade para substratos específicos. (Chen, Sun, Mochly-Rosen, 2010; Marchitti et al., 2008; Song et al., 2011)

O gene que codifica a ALDH2, com 517 peptídeos, está localizado no cromossomo 12q24. Após codificada a enzima é transportada para a matriz mitocondrial em um processo que depende do peptídeo sinal localizado na porção $\mathrm{N}$-terminal. Na matriz mitocondrial ocorre a clivagem do peptídeo sinal (17 peptídeos), permitindo a maturação completa da enzima, com 500 peptídeos formando uma enzima homotetramérica de $\sim 56 \mathrm{kDa}$. Cada subunidade da enzima é composta por três domínios: um domínio catalítico, formado por 3 resíduos de 
cisteína: Cys 301, 302 e 303; um domínio de ligação da coenzima NAD+; e um domínio de oligomerização (Chen et al., 2014; Gross et al., 2015).

A ALDH2 é expressa abundantemente no fígado e pulmão, e está também presente em órgãos que requerem uma capacidade mitocondrial elevada para a geração de ATP, tais como, coração e cérebro (Marchitti et al., 2008). A ALDH2 é também conhecida pelo seu papel em remover o acetaldeído, produzido durante o metabolismo do etanol pela álcool desidrogenase. A ALDH2 é responsável pelo metabolismo de aldeídos alifáticos de cadeia curta, sendo responsável pela conversão oxidativa do 4-hidroxinonenal (4-HNE) ao seu ácido fraco, o ácido 4hidroxinonenóico (4-HNA). Em adição à sua atividade de desidrogenase, a ALDH2 possui atividade de redutase, na conversão da nitroglicerina em óxido nítrico e atividade de esterase na hidrólise da nitroglicerina para gerar o 1,2-dinitrato de gliceril e nitrato (Li et al., 2006).

Além disso, a ALDH2 é conhecida por apresentar uma mutação pontual, $\mathrm{ALDH}^{\star}{ }^{*}$, presente em $40 \%$ da população asiática. Essa mutação afeta cerca de $8 \%$ da população mundial, ou aproximadamente 560 milhões de pessoas, sendo a mutação mais prevalente em humanos (Gross et al., 2015). A mutação ALDH2*2 é decorrente de uma substituição pontual de nucleotídeos (G para A) no éxon 12, que resulta na troca do ácido glutâmico (Glu, E) por uma lisina (Lys, K) na região 487 da ALDH2, mutação também chamada por E487K. Essa troca promove uma alteração na estrutura tridimensional da enzima e resulta na desestabilização do sítio de ligação do cofator $\mathrm{NAD}^{+}$, com consequente diminuição da atividade enzimática em até $95 \%$.

Como podemos observar na figura 2, a estrutura cristalográfica da ALDH2 WT (Protein Data Bank Acession Code PDB:1005) mostra os resíduos 245-262, que formam a hélice aG (roxo), e os resíduos 466-478, que formam o loop do sítio ativo (azul). Na enzima ALDH2*2 mutante (PDB: 1ZUM), a substituição E487K resulta na perda de densidade de elétrons nas duas regiões. A ativação seletiva da ALDH2, utilizando uma pequena molécula (N-(1,3-benzodioxol-5-dylmethyl)-2,6dichlorobenzamide) desenvolvida pelos nossos colaboradores de Stanford, chamada Alda-1 (aldehyde dehydrogenase activator 1), se liga na enzima ALDH2*2 (vermelho - PDB: 3INL), restaurando, de forma alostérica, a estrutura da a hélice (roxo) e do 
loop (azul) para próximo da enzima WT (Larson, Weiner, Hurley, 2005; Larson et al., 2007; Perez-Miller et al., 2010).
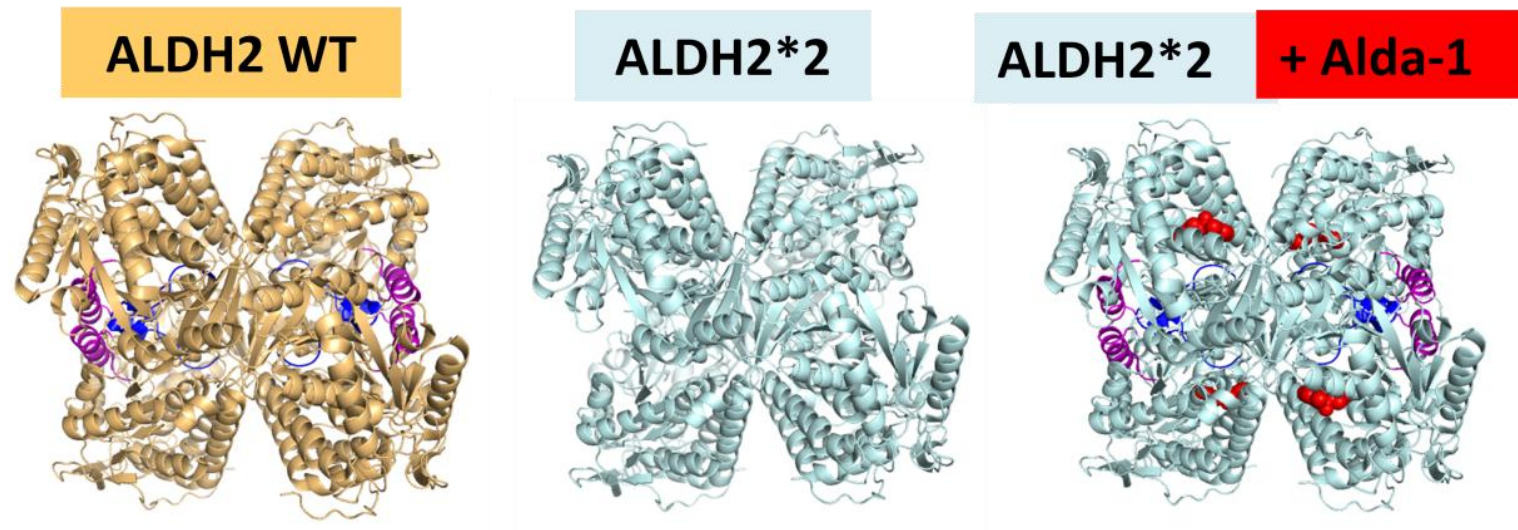

Figura 2 - Estrutura cristalográfica da enzima ALDH2 WT selvagem, ALDH2*2 mutante e da ALDH2*2 mutante com a molécula ativadora Alda-1.

Portadores dessa mutação $\mathrm{ALDH} 2^{*} 2$ são geralmente reconhecidos pela intolerância ao álcool; porém, estes também apresentam maior probabilidade em desenvolver diferentes tipos de câncer (Brooks et al., 2009; Chen et al., 2006; Seitz, Stickel, 2010) e doenças degenerativas (Budas, Disatnik, Mochly-Rosen, 2009; Chen et al., 2008).

Demonstramos, recentemente, que tanto na progressão da cardiomiopatia induzida por infarto do miocárdio quanto na insuficiência cardíaca ocorre uma disfunção mitocondrial e geração de espécies reativas de oxigênio (EROS), peroxidação lipídica e aumento exacerbado na produção de aldeídos tóxicos com consequente diminuição da atividade enzimática da ALDH2. Além disso, vimos que a ativação seletiva da ALDH2, utilizando a molécula ativadora da ALDH2, Alda-1, foi capaz de reduzir a quantidade de aldeídos tóxicos cardíacos, restaurar o metabolismo mitocondrial e melhorar a função cardíaca (Gomes et al., 2014). Ainda, demonstramos que a Alda-1 tem efeito protetor em modelo de isquemia cardíaca in vivo (Sun, Ferreira, Mochly-Rosen, 2011).

Considerando os resultados supracitados mostrando o importante valor da ALDH2 na manutenção da viabilidade cardíaca perante estímulos estressores, decidimos avaliar no presente estudo se camundongos que possuem a mutação $\mathrm{ALDH}^{*} 2$, e consequente atividade da ALDH2 reduzida, são mais suscetíveis ao 
desenvolvimento da insuficiência cardíaca induzida por infarto do miocárdio; e se a molécula Alda-1 é capaz de minimizar a degeneração cardíaca observada na IC. 


\section{CONCLUSÃO}

A mutação E487K da ALDH2 reduz a atividade da enzima ALDH2 e induz um déficit metabólico em mitocôndria isolada, e um estresse redox observado pela menor razão glutationa reduzida/oxidada e pelo acúmulo de adutos 4-HNE-proteína. No entanto, essa condição não altera a função cardíaca dos animais. Mesmo frente ao estresse causado pelo infarto do miocárdio, os animais heterozigotos e homozigotos desenvolvem a mesma disfunção cardíaca que os animais selvagens.

Além disso, a Alda-1 foi efetiva em restaurar os danos cardíacos oriundos do infarto do miocárdio, recuperando a função cardíaca, tanto nos animais selvagens, quanto nos mutantes heterozigotos e homozigotos. No entanto, quando avaliamos a função mitocondrial, os mutantes parecem já ter uma proteção intrínseca com respiração basal e liberação de peróxido mais baixa que o WT.

Baseado nisso, nossos resultados fornecem evidências de que a ALDH2 é uma importante enzima que auxilia na manutenção da viabilidade cardíaca pósinfarto do miocárdio, e que a redução da carga de aldeídos cardíacos através da ativação seletiva ALDH2 é suficiente para atenuar danos causados pela insuficiência cardíaca. 


\section{REFERÊNCIAS*}

Abel ED, Doenst T. Mitochondrial adaptations to physiological vs. pathological cardiac hypertrophy. Cardiovasc Res. 2011;90(2):234-42.

Abramov AY, Gegg M, Grunewald A, Wood NW, Klein C, Schapira AH. Bioenergetic consequences of PINK1 mutations in Parkinson disease. PLoS One. $2011 ; 6(10): \mathrm{e} 25622$.

Antico Arciuch VG, Elguero ME, Poderoso JJ, Carreras MC. Mitochondrial regulation of cell cycle and proliferation. Antioxid Redox Signal. 2012;16(10):1150-80.

Backos DS, Fritz KS, Roede JR, Petersen DR, Franklin CC. Posttranslational modification and regulation of glutamate-cysteine ligase by the $\alpha, \beta$-unsaturated aldehyde 4-hydroxy-2-nonenal. Free Radic Biol Med. 2011;50(1):14-26.

Belch JJ, Bridges AB, Scott N, Chopra M. Oxygen free radicals and congestive heart failure. Br Heart J. 1991;65(5):245-8.

Bradford MM. A rapid and sensitive method for the quantitation of microgram quantities of protein utilizing the principle of protein-dye binding. Anal Biochem. 1976;72:248-54.

Brookes PS, Yoon Y, Robotham JL, Anders MW, Sheu SS. Calcium, ATP, and ROS: a mitochondrial love-hate triangle. Am J Physiol Cell Physiol. 2004;287(4):C817-33.

Brooks PJ, Enoch MA, Goldman D, Li TK, Yokoyama A. The alcohol flushing response: an unrecognized risk factor for esophageal cancer from alcohol consumption. PLoS Med. 2009;6(3):e50.

Budas GR, Disatnik MH, Mochly-Rosen D. Aldehyde dehydrogenase 2 in cardiac protection: a new therapeutic target? Trends Cardiovasc Med. 2009;19(5):158-64.

Campos JC, Queliconi BB, Dourado PM, Cunha TF, Zambelli VO, Bechara LR, et al. Exercise training restores cardiac protein quality control in heart failure. PLoS One. 2012;7(12):e52764.

Cancherini DV, Queliconi BB, Kowaltowski AJ. Pharmacological and physiological stimuli do not promote $\mathrm{Ca}(2+)$-sensitive $\mathrm{K}+$ channel activity in isolated heart mitochondria. Cardiovasc Res. 2007;73(4):720-8.

Chen CH, Budas GR, Churchill EN, Disatnik MH, Hurley TD, Mochly-Rosen D. Activation of aldehyde dehydrogenase-2 reduces ischemic damage to the heart. Science. 2008;321(5895):1493-5.

*De acordo com:

International Committee of Medical Journal Editors. [Internet]. Uniform requirements for manuscripts submitted to biomedical journals. [2011 Jul 15]. Available from: 
http://www.nlm.nih.gov/bsd/uniform_requirements.htlm

Chen $\mathrm{CH}$, Ferreira JC, Gross ER, Mochly-Rosen D. Targeting aldehyde dehydrogenase 2: new therapeutic opportunities. Physiol Rev. 2014;94(1):1-34.

Chen $\mathrm{CH}$, Sun L, Mochly-Rosen D. Mitochondrial aldehyde dehydrogenase and cardiac diseases. Cardiovasc Res. 2010;88(1):51-7.

Chen YJ, Chen C, Wu DC, Lee $\mathrm{CH}$, Wu Cl, Lee JM, et al. Interactive effects of lifetime alcohol consumption and alcohol and aldehyde dehydrogenase polymorphisms on esophageal cancer risks. Int J Cancer. 2006;119(12):2827-31.

Chen ZH, Niki E. 4-hydroxynonenal (4-HNE) has been widely accepted as an inducer of oxidative stress. Is this the whole truth about it or can 4-HNE also exert protective effects? IUBMB Life. 2006;58(5-6):372-3.

Colucci WS. The effects of norepinephrine on myocardial biology: implications for the therapy of heart failure. Clin Cardiol. 1998;21(12 Suppl 1):I20-4.

da Silva MM, Sartori A, Belisle E, Kowaltowski AJ. Ischemic preconditioning inhibits mitochondrial respiration, increases $\mathrm{H} 2 \mathrm{O} 2$ release, and enhances $\mathrm{K}+$ transport. Am J Physiol Heart Circ Physiol. 2003;285(1):H154-62.

Dhalla AK, Hill MF, Singal PK. Role of oxidative stress in transition of hypertrophy to heart failure. J Am Coll Cardiol. 1996;28(2):506-14.

Di Lisa F, Kaludercic N, Carpi A, Menabò R, Giorgio M. Mitochondria and vascular pathology. Pharmacol Rep. 2009;61(1):123-30.

Doser TA, Turdi S, Thomas DP, Epstein PN, Li SY, Ren J. Transgenic overexpression of aldehyde dehydrogenase-2 rescues chronic alcohol intakeinduced myocardial hypertrophy and contractile dysfunction. Circulation. 2009;119(14):1941-9.

Endo J, Sano M, Katayama T, Hishiki T, Shinmura K, Morizane S, et al. Metabolic remodeling induced by mitochondrial aldehyde stress stimulates tolerance to oxidative stress in the heart. Circ Res. 2009;105(11):1118-27.

Facundo HdTF. Efeitos redox e protetores do pré-condicionamento isquêmico e da abertura do canal mitocondrial de potássio sensível a ATP contra morte celular por isquemia e reperfusão cardíaca. Tese (Doutorado em Ciências Bioquímica). São Paulo: Instituto de Química, Universidade de São Paulo; 2007. 94 p.

Farrés J, Wang X, Takahashi K, Cunningham SJ, Wang TT, Weiner H. Effects of changing glutamate 487 to lysine in rat and human liver mitochondrial aldehyde dehydrogenase. A model to study human (Oriental type) class 2 aldehyde dehydrogenase. J Biol Chem. 1994;269(19):13854-60. 
Ferencz-Biro K, Pietruszko R. Human aldehyde dehydrogenase: catalytic activity in oriental liver. Biochem Biophys Res Commun. 1984;118(1):97-102.

Ferreira JC, Boer BN, Grinberg M, Brum PC, Mochly-Rosen D. Protein quality control disruption by PKC $\beta I I$ in heart failure; rescue by the selective PKC $\beta$ II inhibitor, $\beta$ IIV53. PLoS One. 2012;7(3):e33175.

Ferreira JC, Brum PC, Mochly-Rosen D. BIIPKC and $\varepsilon P K C$ isozymes as potential pharmacological targets in cardiac hypertrophy and heart failure. $\mathrm{J}$ Mol Cell Cardiol. $2011 ; 51(4): 479-84$.

Ferreira JC, Koyanagi T, Palaniyandi SS, Fajardo G, Churchill EN, Budas G, et al. Pharmacological inhibition of $\beta$ IIPKC is cardioprotective in late-stage hypertrophy. $J$ Mol Cell Cardiol. 2011;51(6):980-7.

Ferreira JC, Mochly-Rosen D. Nitroglycerin use in myocardial infarction patients. Circ J. 2012;76(1):15-21.

Ferreira JC, Rolim NP, Bartholomeu JB, Gobatto CA, Kokubun E, Brum PC. Maximal lactate steady state in running mice: effect of exercise training. Clin Exp Pharmacol Physiol. 2007;34(8):760-5.

Fiorelli AI, Coelho GHB, Oliveira Junior JdL, Oliveira AS. Insuficiência cardíaca e transplante cardíaco. Revista Medicina2008. p. 105-20.

Frederick DW, Loro E, Liu L, Davila A, Chellappa K, Silverman IM, et al. Loss of NAD Homeostasis Leads to Progressive and Reversible Degeneration of Skeletal Muscle. Cell Metab. 2016;24(2):269-82.

Gomes KM, Bechara LR, Lima VM, Ribeiro MA, Campos JC, Dourado PM, et al. Aldehydic load and aldehyde dehydrogenase 2 profile during the progression of postmyocardial infarction cardiomyopathy: benefits of Alda-1. Int J Cardiol. 2015;179:12938.

Gomes KM, Campos JC, Bechara LR, Queliconi B, Lima VM, Disatnik MH, et al. Aldehyde dehydrogenase 2 activation in heart failure restores mitochondrial function and improves ventricular function and remodelling. Cardiovasc Res. 2014;103(4):498-508.

Gross ER, Zambelli VO, Small BA, Ferreira JC, Chen CH, Mochly-Rosen D. A personalized medicine approach for Asian Americans with the aldehyde dehydrogenase 2*2 variant. Annu Rev Pharmacol Toxicol. 2015;55:107-27.

Gupta S, Prahash AJ, Anand IS. Myocyte contractile function is intact in the postinfarct remodeled rat heart despite molecular alterations. Cardiovasc Res. 2000;48(1):77-88.

Holt E, Tønnessen T, Lunde PK, Semb SO, Wasserstrom JA, Sejersted OM, et al. Mechanisms of cardiomyocyte dysfunction in heart failure following myocardial infarction in rats. J Mol Cell Cardiol. 1998;30(8):1581-93. 
Houser SR, Margulies KB. Is depressed myocyte contractility centrally involved in heart failure? Circ Res. 2003;92(4):350-8.

Kaplan P, Tatarkova Z, Racay P, Lehotsky J, Pavlikova M, Dobrota D. Oxidative modifications of cardiac mitochondria and inhibition of cytochrome c oxidase activity by 4-hydroxynonenal. Redox Rep. 2007;12(5):211-8.

Kowaltowski AJ, de Souza-Pinto NC, Castilho RF, Vercesi AE. Mitochondria and reactive oxygen species. Free Radic Biol Med. 2009;47(4):333-43.

Kutuk O, Basaga $\mathrm{H}$. Apoptosis signalling by 4-hydroxynonenal: a role for JNK-cJun/AP-1 pathway. Redox Rep. 2007;12(1):30-4.

Lai CL, Yao CT, Chau GY, Yang LF, Kuo TY, Chiang CP, et al. Dominance of the inactive Asian variant over activity and protein contents of mitochondrial aldehyde dehydrogenase 2 in human liver. Alcohol Clin Exp Res. 2014;38(1):44-50.

Lang RM, Bierig M, Devereux RB, Flachskampf FA, Foster E, Pellikka PA, et al. Recommendations for chamber quantification: a report from the American Society of Echocardiography's Guidelines and Standards Committee and the Chamber Quantification Writing Group, developed in conjunction with the European Association of Echocardiography, a branch of the European Society of Cardiology. J Am Soc Echocardiogr. 2005;18(12):1440-63.

Larson HN, Weiner H, Hurley TD. Disruption of the coenzyme binding site and dimer interface revealed in the crystal structure of mitochondrial aldehyde dehydrogenase "Asian" variant. J Biol Chem. 2005;280(34):30550-6.

Larson HN, Zhou J, Chen Z, Stamler JS, Weiner H, Hurley TD. Structural and functional consequences of coenzyme binding to the inactive asian variant of mitochondrial aldehyde dehydrogenase: roles of residues 475 and 487 . J Biol Chem. 2007;282(17):12940-50.

Li P, Park C, Micheletti R, Li B, Cheng W, Sonnenblick EH, et al. Myocyte performance during evolution of myocardial infarction in rats: effects of propionyl-Lcarnitine. Am J Physiol. 1995;268(4 Pt 2):H1702-13.

Li Y, Zhang D, Jin W, Shao C, Yan P, Xu C, et al. Mitochondrial aldehyde dehydrogenase-2 (ALDH2) Glu504Lys polymorphism contributes to the variation in efficacy of sublingual nitroglycerin. J Clin Invest. 2006;116(2):506-11.

Liu L, Eisen HJ. Epidemiology of heart failure and scope of the problem. Cardiology clinics. 2014;32(1):1-8, vii.

Liu T, O'Rourke B. Regulation of mitochondrial $\mathrm{Ca} 2+$ and its effects on energetics and redox balance in normal and failing heart. $\mathrm{J}$ Bioenerg Biomembr. 2009;41(2):127-32. 
Loennechen JP, Wisløff U, Falck G, Ellingsen $\varnothing$. Cardiomyocyte contractility and calcium handling partially recover after early deterioration during post-infarction failure in rat. Acta Physiol Scand. 2002;176(1):17-26.

Ma H, Guo R, Yu L, Zhang Y, Ren J. Aldehyde dehydrogenase 2 (ALDH2) rescues myocardial ischaemia/reperfusion injury: role of autophagy paradox and toxic aldehyde. Eur Heart J. 2011;32(8):1025-38.

Ma H, Yu L, Byra EA, Hu N, Kitagawa K, Nakayama Kl, et al. Aldehyde dehydrogenase 2 knockout accentuates ethanol-induced cardiac depression: role of protein phosphatases. J Mol Cell Cardiol. 2010;49(2):322-9.

Mackiewicz U, Maczewski M, Konior A, Tellez JO, Nowis D, Dobrzynski H, et al. Sarcolemmal $\mathrm{Ca} 2+-$ ATPase ability to transport $\mathrm{Ca} 2+$ gradually diminishes after myocardial infarction in the rat. Cardiovasc Res. 2009;81(3):546-54.

Mallat Z, Philip I, Lebret M, Chatel D, Maclouf J, Tedgui A. Elevated levels of 8-isoprostaglandin F2alpha in pericardial fluid of patients with heart failure: a potential role for in vivo oxidant stress in ventricular dilatation and progression to heart failure. Circulation. 1998;97(16):1536-9.

Marchitti SA, Brocker C, Stagos D, Vasiliou V. Non-P450 aldehyde oxidizing enzymes: the aldehyde dehydrogenase superfamily. Expert Opin Drug Metab Toxicol. 2008;4(6):697-720.

Mitchell P, Moyle J. Chemiosmotic hypothesis of oxidative phosphorylation. Nature. 1967;213(5072):137-9.

Nakamura K, Kusano K, Nakamura Y, Kakishita M, Ohta K, Nagase S, et al. Carvedilol decreases elevated oxidative stress in human failing myocardium. Circulation. 2002;105(24):2867-71.

Nojiri H, Shimizu T, Funakoshi M, Yamaguchi O, Zhou H, Kawakami S, et al. Oxidative stress causes heart failure with impaired mitochondrial respiration. J Biol Chem. 2006;281(44):33789-801.

Ott M, Gogvadze V, Orrenius S, Zhivotovsky B. Mitochondria, oxidative stress and cell death. Apoptosis. 2007;12(5):913-22.

Palaniyandi SS, Qi X, Yogalingam G, Ferreira JC, Mochly-Rosen D. Regulation of mitochondrial processes: a target for heart failure. Drug Discov Today Dis Mech. 2010;7(2):e95-e102.

Perez-Miller S, Younus H, Vanam R, Chen CH, Mochly-Rosen D, Hurley TD. Alda-1 is an agonist and chemical chaperone for the common human aldehyde dehydrogenase 2 variant. Nat Struct Mol Biol. 2010;17(2):159-64.

Pfeffer MA, Pfeffer JM, Fishbein MC, Fletcher PJ, Spadaro J, Kloner RA, et al. Myocardial infarct size and ventricular function in rats. Circ Res. 1979;44(4):503-12. 
Poyton RO, Ball KA, Castello PR. Mitochondrial generation of free radicals and hypoxic signaling. Trends Endocrinol Metab. 2009;20(7):332-40.

Rahman I, Kode A, Biswas SK. Assay for quantitative determination of glutathione and glutathione disulfide levels using enzymatic recycling method. Nat Protoc. 2006;1(6):3159-65.

SBC. Atualização da Diretriz Brasileira de Insuficiência Cardíaca Crônica, 2012. 2012:http://publicacoes.cardiol.br/consenso/2012/Diretriz\%20IC\%20Cr\%C3\%B4nica. pdf.

Seitz HK, Stickel F. Acetaldehyde as an underestimated risk factor for cancer development: role of genetics in ethanol metabolism. Genes Nutr. 2010;5(2):121-8.

Singh N, Dhalla AK, Seneviratne C, Singal PK. Oxidative stress and heart failure. Mol Cell Biochem. 1995;147(1-2):77-81.

Song BJ, Abdelmegeed MA, Yoo SH, Kim BJ, Jo SA, Jo I, et al. Post-translational modifications of mitochondrial aldehyde dehydrogenase and biomedical implications. J Proteomics. 2011;74(12):2691-702.

Song J, Zhang XQ, Wang J, Carl LL, Ahlers BA, Rothblum LI, et al. Sprint training improves contractility in postinfarction rat myocytes: role of $\mathrm{Na}+\mathrm{Ca} 2+$ exchange. $\mathrm{J}$ Appl Physiol (1985). 2004;97(2):484-90.

Sun L, Ferreira JC, Mochly-Rosen D. ALDH2 activator inhibits increased myocardial infarction injury by nitroglycerin tolerance. Sci Transl Med. 2011;3(107):107ra11.

Tahara EB, Navarete FD, Kowaltowski AJ. Tissue-, substrate-, and site-specific characteristics of mitochondrial reactive oxygen species generation. Free Radic Biol Med. 2009;46(9):1283-97.

Tingberg E, Ohlin AK, Gottsäter A, Ohlin H. Lipid peroxidation is not increased in heart failure patients on modern pharmacological therapy. Int $\mathrm{J}$ Cardiol. 2006;112(3):275-81.

Tsutsui $\mathrm{H}$, Kinugawa S, Matsushima S. Oxidative stress and heart failure. Am J Physiol Heart Circ Physiol. 2011;301(6):H2181-90.

Van Klinken JB, van den Berg SA, Havekes LM, Willems Van Dijk K. Estimation of activity related energy expenditure and resting metabolic rate in freely moving mice from indirect calorimetry data. PLoS One. 2012;7(5):e36162.

Ventura-Clapier R, Garnier A, Veksler V, Joubert F. Bioenergetics of the failing heart. Biochim Biophys Acta. 2011;1813(7):1360-72.

Wang C, Fan F, Cao Q, Shen C, Zhu H, Wang P, et al. Mitochondrial aldehyde dehydrogenase 2 deficiency aggravates energy metabolism disturbance and diastolic dysfunction in diabetic mice. J Mol Med (Berl). 2016. 
WHO. World Health Organization. Global status report on noncommunicable diseases. 2014

Wisløff U, Loennechen JP, Currie S, Smith GL, Ellingsen $\varnothing$. Aerobic exercise reduces cardiomyocyte hypertrophy and increases contractility, Ca2+ sensitivity and SERCA-2 in rat after myocardial infarction. Cardiovasc Res. 2002;54(1):162-74.

Yang Y, Sharma R, Sharma A, Awasthi S, Awasthi YC. Lipid peroxidation and cell cycle signaling: 4-hydroxynonenal, a key molecule in stress mediated signaling. Acta Biochim Pol. 2003;50(2):319-36.

Young-Collier KJ, McArdle M, Bennett JP. The dying of the light: mitochondrial failure in Alzheimer's disease. J Alzheimers Dis. 2012;28(4):771-81.

Zhang Y, Babcock SA, Hu N, Maris JR, Wang H, Ren J. Mitochondrial aldehyde dehydrogenase (ALDH2) protects against streptozotocin-induced diabetic cardiomyopathy: role of GSK3 $\beta$ and mitochondrial function. BMC Med. 2012;10:40. 